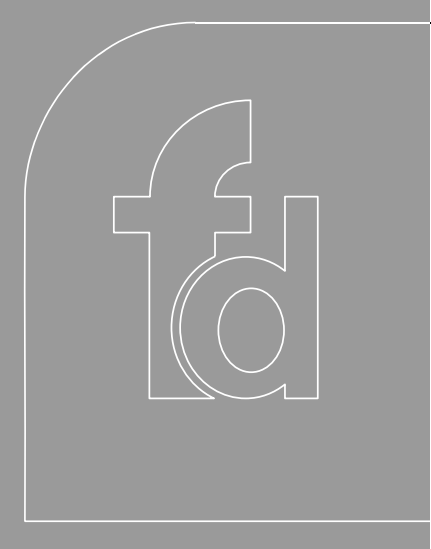

CONTENTS

MALDI-MS: ideal for high-throughput proteomics?

Proteomic analysis by MALDI-MS \& -MS/MS

Derivatization of proteins \& peptides

Protein sample preparation for MALDI-MS

Improvements of the MALDI process

MALDI-MS pattem diagnostics

Imaging MS

Summary \& conclusion

Expert commentary

Five-yearview

Key issues

References

Affiliations

${ }^{\dagger}$ Author for correspondence The BioC entre, University of Reading, Whiteknights, PO Box 221, Reading

RG 6 6AS, UK

Tel.: +44 1183784552

Fax: +44 1183784551

r.k.cramer@rdg.ac.uk

KEYWORDS:

2DE, automation,

high-throughput, liquid

chromatography, liquid matrices,

M ALDI, mass spectrometry,

peptide derivatization,

proteomics, robotics

\title{
High-throughput proteomics using matrix- assisted laser desorption/ ionization mass spectrometry
}

\author{
Rainer $\mathrm{Cramer}^{\dagger}$, J ohan Gobom and Eckhard Nordhoff
}

It has become evident that the mystery of life will not be deciphered just by decoding its blueprint, the genetic code. In the life and biomedical sciences, research efforts are now shifting from pure gene analysis to the analysis of all biomolecules involved in the machinery of life. One area of these postgenomic research fields is proteomics. Although proteomics, which basically encompasses the analysis of proteins, is nota new concept, it is far from being a research field that can rely on routine and large-scale analyses. At the time the tem proteomics was coined, a gold-rush mentality was created, promising vast and quick riches (i.e., solutions to the immensely complex questions of life and disease). Predictably, the reality has been quite different The complexity of proteomes and the wide variations in the abundances and chemical properties of their constituents has rendered the use of systematic analytical approaches only partially successful, and biologically meaningful results have been slow to amive. However, to leam more about how cells and, hence, life works, it is essential to understand the proteins and their complex interactions in theirnative environment This is why proteomics will be an important partof the biomedical sciences for the foreseeable future. Therefore, any advances in providing the tools that make protein analysis a more routine and large-scale business, ideally using automated and rapid analytical procedures, are highly sought after. This review will provide some basics, thoughts and ideas on the exploitation of matrix-assisted laser desorption/ ionization in biological mass spectrometry - one of the most commonly used analytical tools in proteomics - for high-throughput analyses.

Expert Rev. Proteomics 2(3), 407-420 (2005)

While protein analysis is a bioanalytical field older than DNA/RN A analysis, the term proteomics is, in contrast, much younger than genomics. Although proteins were analyzed long before D N A and RN A, it was only in the 1990s that the advent of electrospray ionization (ESI) and matrix-assisted laser desorption/ionization (MALDI) in biological mass spectrometry (MS) opened the door to rapid and highly sensitive protein analysis. Since then, proteomics has progressed from the initial concept stage to large-scale analyses of proteomes. However, one of the initial promises, high-throughput (HTP) proteomics, is a long way from being realized.

Recent technological advances in mass analyzers [1-5] clearly demonstrate that the mass spectrometric instrumentation is largely in place for HTP proteomics [6,7]. Nonetheless, there are still major obstacles to successful HTP proteomics. These are mainly associated with peptide/protein separation, purification and recovery as well as the vast complexity of post-translational isoforms and their intriguing interactions, which place high demands on data analysis. This review is concerned with the utilization of MALDI-MS in HTP proteomics. Since the requirements in post-M S data processing are less dependant on the different M S techniques than the pre-M S sample preparation requirements and the MS technique itself, this review will focus on the latter with respect to MALDI-MS and its position in HTP proteomics. MALDI-MS data handling and mining in proteomics will consequently not be discussed. However, in the 
overall proteomic workflow, the area of MS data handling and mining has not yet been adequately addressed, but is currently under discussion and is immensely important for H TP analysis [8-14].

In this review, some of the basic principles and requirements of HTP proteomics with respect to the employment of M ALDI-M S are presented. Effort has been made in this review to avoid contributing to the hype and hope in proteomics by discussing concepts and ideas rather than stating numbers, which are often uninformative or even deceptive without providing their context in detail. H ence, particulars about HTP platforms either commercially available or built in research laboratories have not been included.

\section{MALDI-MS: ideal for high-throughput proteomics?}

MALDI [15-18] is arguably the mass spectrometric ionization technique of choice for HTP and high-sensitivity analysis of peptides and proteins [19]. With a single laser shot, an entire mass spectrum can be acquired within microseconds in a timeof-flight (TOF) mass spectrometer [20]. This type of mass analyzer is also one of the most sensitive due to its high ion transmission. In addition, MALDI-TOF technology is inherently simple and TOF mass spectrometers can be produced as lowcost analyzers. In contrast to ESI (the only serious alternative soft ionization technique), MALDI data acquisition can be stopped at any point and unconsumed sample recovered for later analysis. Furthermore, M ALDI is more tolerant to sample contaminations such as salt and buffers. All these advantages are well known and already make M ALDI-M S the technique of choice for H TP proteome analysis in many research laboratories.

$\mathrm{H}$ owever, there are disadvantages when compared with ESI $[21,22]$. To date, the most succesfful M ALDI technique in proteomics is based on pulsed ultraviolet (UV) laser irradiation and sample preparations that lead to matrix crystals incorporating the analyte molecule. There are major implications for its analytical performance arising from this. Crystalline MALD I samples are notoriously inhomogeneous, leading to highly fluctuating analyte ion signals from one laser shot to another, particularly when the laser desorption spot is moved to another location. As a consequence, typically both the laser irradiance and desorption spot need to be frequently changed during data acquisition, rendering fully automated analysis, a prerequisite for high sample throughput, difficult and time consuming. In addition, UV-M ALDI is in fact an ablation process (typically in the $100 \mathrm{~nm}$ range) and any changes in the sample spot morphology, such as through the M ALDI process itself (or desorption spot changes), lead to significant alterations of the extraction geometry. Thus, instrumental parameters for optimal ion extraction and transmission lose their validity, resulting in potentially poorer mass accuracy, resolution and ion detection.

In contrast, ESI has inherently higher sample homogeneity and analyte ion flux stability due to its sample's liquidity. These characteristics allow easier interfacing to ion flux-sensitive instruments such as Fourier transform ion cyclotron resonance (FTICR) mass spectrometers and potentially quantification using ion signal intensities alone [23,24]. In addition, liquid chromatography (LC) can be directly coupled to ESI ion sources, enabling automated workflows with peptide/protein separation and purification $[25,26]$.

N onetheless, the important characteristics of H TP platforms such as ease, speed, robustness and low cost of theinstrumentation are met by M ALDI rather than ESI.

\section{Proteomic analysis by MALDI-MS \&-MS/MS}

$O$ ne of the main reasons why M S has become a key technique in proteome research is its ability to identify and characterize endogenous proteins isolated from small cell or tissue samples using large sequence databases. In this section, a quick overview of the two main protein identification strategies using M ALDIMS and tandem MS (M S/MS) is provided. In recent years, these strategies have been the subject of extensive development for H T P capacity.

\section{Protein identification by MALDI-MS peptide mass mapping}

M ALDI peptide mass mapping (PM M ) is a widely used technique for identification of proteins $[7,27,28]$. The unknown protein is usually digested by a protease of high specificity, most commonly trypsin, which cleaves polypeptides at the $\mathrm{C}$-terminal side of lysine and arginine residues, unless the next amino acid in the sequence is proline. MALDI-MS analysis of the resulting mixture of peptides yields a peptide mass fingerprint (PM F), which is a set of measured molecular masses of proteolytic peptide ions obtained from the protein digest. In a database search, these mass values are matched against sets of proteolytic peptide masses calculated for all protein sequences in a sequence database. The search returns a list of the database entries with the highest number of matching peptide mass values, and various al gorithms are used to rank the sequences and determine the probability that the highest ranking sequence entry is the true identity of the analyzed protein. Recording a PM F instead of simply measuring the molecular mass of the intact protein has several advantages. For example, if a protein carries post-translational modifications, contains mutations or is processed, its measured molecular mass will not match the calculated mass based on its sequence in the database and consequently its identification will fail. The same will happen if the protein's sequence in the database contains an error. In contrast, with a PM F, only signals corresponding to the peptides that carry the modified amino acid, or containing the mutation site, will be affected. The recorded mass-to-charge ratio $(\mathrm{m} / \mathrm{z})$ values of the other signals will match the masses calculated based on the protein's amino acid sequence, thereby maintaining a robust identification procedure, particularly if many different peptides are generated and detected. In addition, the detection sensitivity and mass accuracy are significantly higher for peptides than for proteins. However, protein identification using PM Fs relies on the fact that the detected peptides are derived from a single protein. If more than one protein is present in the sample, the confidence of the search results decreases considerably. Therefore, the technique is most often used in 
combination with high-specificity affinity purification techniques or high-resolving protein separation techniques such as 2D gel electrophoresis (2D E) [29,30].

\section{Protein identification by MALDI-MS/MS}

MALDI-MS is not limited to measuring the masses of intact analyte molecules. $M$ any instruments provide the possibility of isolating a selected analyte ion and, by different activation methods, transfer energy to it, thereby inducing fragmentation reactions. $M$ ass analysis of the resulting fragment ions can provide detailed structural information about the analyte and is termed MS/MS analysis. On MALDI-TOF mass spectrometers, analyte fragmentation is often induced by increasing the laser energy above the desorption threshold. Under these conditions, the fragmentation process is typically delayed such that most of the fragment ions are formed outside the ion source in the field-free drift region. Accordingly, these events are termed post-source decay (PSD) [31,32].

There are several strategies for protein identification using M S/M S data [33]. Analogously to viewing a PM $F$ as a fingerprint of the analyzed protein, a fragment ion spectrum can be regarded as a fingerprint of the fragmented peptide, and thus be used directly for protein identification in sequence databases. In this strategy, the fragment ion masses recorded for one selected pre cursor peptide molecular ion are compared with the sets of possible fragment ion masses calculated for each proteolytic peptide sequence, which matches the mass of the selected peptide ion, for each protein sequence in the database. Another strategy for protein identification is to generate sufficient sequence information from individual cleavage products of the protein to identify it in a sequence database. This is achieved by interpretation of the fragment ion spectrum (e.g., by assigning $\mathrm{m} / \mathrm{z}$ distances between signals to single amino acid residues and often known as de novo sequencing). Successful de novo sequencing requires spectra of high quality, ideally containing contiguous signal series representing the same ion type. However, PSD fragment ion spectra often contain signals of many types of fragment ions from different fragmentation reactions, rendering their interpretation difficult. Furthermore, the types and numbers of fragment ions detected depend strongly on the amino acid sequence of the analyzed peptide. Therefore, while de novo sequencing using M ALD I-M S/M S data is a potentially powerful technique, it is not yet routinely applicable to protein identification. Alternatively, when the sequence information only comprises two to five amino acids, which is most often insufficient for identification, it can be combined with the molecular mass of the peptide, the cleavage specificity of the protease used and the distance (in mass) of the determined sequence stretch from the peptide termini to form a socalled peptide sequence tag. A peptide sequence tag constitutes highly specific input for database searches.

However, recent years have seen the development of MALDI-TOF/TOF mass spectrometers with advanced M S/M S capabilities [34-36]. Compared with PSD fragment ion analysis on a conventional MALDI-TOF instrument, spectra acquisition time on M ALDI-TOF/TOF instruments is shorter by at least one order of magnitude, and sample consumption lower to the same extent. In combination with higher repetition rate lasers, several thousand MS/MS spectra can be recorded per day on a single instrument. 0 ne additional advantage of higher repetition lasers, such as solid-state lasers, is the higher lifetime of the laser itself when compared with the nitrogen lasers typically used in M ALDI-MS. In particular, newly developed diode-pumped solid-state lasers, which are now able to provide the power required for M ALDI-M S applications, are providing reliable pulsed laser irradiation with up to kilohertz repetition rates and, thus, have great potential to further extend the use of M ALD I-M S for H TP applications.

Some earlier and parallel developments in coupling M ALDI to analyzers, which are usually employed for continuous ion (e.g., ESI) and not pulsed ion (e.g., M ALDI) generation, have greatly extended the use of MALDI-MS/MS in proteomic research. Hybrid mass analyzers such as quadrupole (q)TOF instruments provide excellent mass accuracy and resolution but do not have the analytical sensitivity of TOF or TOF/TOF instruments in simple M S mode [37-39]. However, the data quality and sensitivity that are achievable for M S/M S measurements usually surpasses TOF/TOF instruments for the precursor ions that survive the initial selection process. N onetheless, their employment in H TP proteomics work is limited by the longer time necessary to acquire a peptide mass fingerprint and its associated greater consumption of sample. In addition, many M ALDI ion sources on these instruments require higher laser irradiation, leading to further substantial consumption and, thus, loss of sample.

\section{Derivatization of proteins \& peptides}

O ne obvious subject for analysis improvement is the analyte itself. By manipulating or modifying the analyte to a degree where it will behave in a perfect manner throughout the analytical process and ensuring that these changes do not impair the information sought, enhanced analytical data can often be obtained. Analyte derivatization is not a new concept in M S, but proteomic-specific strategies have only recently been developed. 0 ne of the most important areas is peptide/ protein modification for reliable quantification by M S. Another main area of peptide derivatization is the enhancement of the mass spectrometric data. In both cases, modifications are often employed that are multifunctional and allow experimental procedures for improved sample preparation.

\section{Mass spectrometric quantification}

O ne important aspect in proteomics is the quantification of proteins. Analysis of protein expression (i.e., the rate of protein synthesis and degradation) is essential to identify and understand the cause and effect of many biological pathways and responses. Consequently, mass spectrometric quantification techniques have been developed and play an increasingly important role in proteome analysis. For accurate mass spectrometric quantification, the analyte signal intensity must be related to the signal intensity of a reference compound. Since 
any difference in the chemical structure of the analyte and the reference molecule can affect losses during the sample preparation as well as the ionization yield, analyte and reference molecules should ideally only differ in mass but be virtually identical otherwise. $\mathrm{H}$ ence, peptide tags have been devised that consist of stable isotopes such as ${ }^{13} \mathrm{C},{ }^{15} \mathrm{~N}$ and ${ }^{18} \mathrm{O}$. These are ideal labels as the exchange of one or more ${ }^{12} \mathrm{C},{ }^{14} \mathrm{~N}$ or ${ }^{16} \mathrm{O}$ atoms in the analyte molecules by ${ }^{13} \mathrm{C},{ }^{15} \mathrm{~N}$ or ${ }^{18} \mathrm{O}$ creates a reference compound that is chemically equivalent but has a different molecular mass. Since modern mass spectrometers, including MALDI-MS instruments, are capable of resolving isotopic variants of the same peptide ion, just a few additional neutrons are sufficient to distinguish molecular ion signals of labeled and unlabeled peptide ions, whereas these isotopic changes are not discriminated by any biological or chemical process during sample preparation.

In proteomics, several analytical strategies using stable isotope-incorporated internal reference compounds have been described [40,41]. $O$ da and coworkers reported a method for relative quantification of proteins in two separate yeast cultures [42]. 0 ne culture was grown in a medium highly enriched in ${ }^{15} \mathrm{~N}$, resulting in metabolic incorporation of the stable isotope into all proteins, while the other culture was grown in a medium with a natural relative abundance of the ${ }^{14} \mathrm{~N}$ isotope $(99.6 \%)$. Protein extracts from the two cell cultures were mixed and, after protein separation and proteolytic digestion, analyzed by M S. Based on the signal intensity ratios recorded for the ${ }^{15} \mathrm{~N}$ - and ${ }^{14} \mathrm{~N}$-incorporated tryptic peptides, the relative abundance of each analyzed protein could be determined with high precision.

Pratt and coworkers published a different strategy [43]. Instead of comparing protein abundance in two separate cell populations, a single cell culture was studied, which, at a defined time point, was subjected to a replacement of the growth medium by one of different stable isotope composition. Thus, proteins synthesized after the medium replacement could

\section{Box 1. The Ten Commandments for peptide derivatization for matrix- assisted laser desorption/ionization mass spectrometry.}

1. (Multi-)functionality (e.g., quantification and affinity isolation)

2. Intact ion detection enhancement

3. Fragment ion detection enhancement

4. Simple fragment ion patterns

5. High-yield and fast reactions

6. No side reactions or by-products

7. Low analyte loss

8. Robust and simple protocols

9. Low consumable costs

10. Compatibility with automation

Points 1-4 are usually the reason for derivatization, while 5-10 outline the demands on the proposed chemistry, with points $8-10$ being particularly important for high-throughput applications. be quantitatively distinguished from those al ready existing. By analyzing proteins isolated at defined time points, this method enabled accurate determination of protein turnover rates.

0 ther strategies that have recently been developed employ a chemical or enzymatic reaction to introduce a stable isotope label during or following proteolytic degradation of the proteins. The main advantage of this approach is that it is independent of the protein synthesis and can therefore be applied to any protein extract, including human tissue samples. Another advantage is the potential inclusion of other functional groups as part of the isotope label. These multifunctional labels can then be used to aid the subsequent sample preparation or mass spectrometric analysis. These reactions and their applications are described in the following section.

\section{Improving sample preparation \& ion detection}

A number of strategies have been developed for peptide labeling to enable protein quantification. These strategies exploit the theoretically identical ion behavior (e.g., detectability and fragmentation) of isobaric [44] or isotopically [45,46] different forms of the analyte in mass spectrometers and/or sample purification steps. Samples derived under different conditions can be differentially labeled and then combined for subsequent coanalysis by M S, thus enabling quantification. Some of these strategies also employ affinity labels that provide the means of reducing the sample complexity and increasing the sample purity by selectively enriching the labeled peptides (see below).

0 ther peptide derivatization methods specifically focus on improvements in M ALDI ion detection or fragmentation. Guanidination of lysine residues has been applied to enhance the anaIyte ion signal intensity via increased protonation through higher solution- and gas-phase basicity [47,48]. Similarly, peptide labeling using cationic moieties, leading to a permanent positive charge, has also been reported to increase the peptide ion detection [49]. Recently, coumarin tags have been applied, which suggests that the greater hydrophobicity of the tagged analyte improves peptide incorporation and, thus, the M ALDI process [50].

In contrast, derivatization strategies using strongly acidic functionalities, such as sulfonic acids, usually result in poorer peptide ionization. However, peptide ion fragmentation is greatly enhanced by providing a mobile proton that specifically facilitates peptide amide bond cleavage, resulting in strong $y$-fragment ion series, thus making M ALDI-M S/M S peptide ion sequencing simple and highly sensitive [51-53]. H owever, the poorer peptide ionization is currently being addressed by several groups using improved derivatization protocols [49,54], multifunctionality tags, such as coumarin tags with sulfonic acid moieties [50], or affinity chromatography for selective enrichment of the derivatized peptides [55].

MALDI-MS and -MS/MS will undoubtedly benefit from further advances in peptide derivatization schemes. Although many requirements have to be addressed (BOX 1), the future potential and advantages are promising, as the MALDILIFT/PSD -M S/M S spectra of derivatized peptides in FIGURE 1 clearly demonstrate. 


\section{Protein sample preparation for MALI-MS}

For the analysis of proteomes, one goal is to separate and identify as many protein species as possible in a proteome extract or fraction thereof. Protein samples need to be simplified and prepared to an acceptable level for M S analysis. Therefore, protein sample preparation including the separation, purification and selective enrichment of proteins or their proteolytic products is a pivotal requirement for succesfful proteomic analysis.
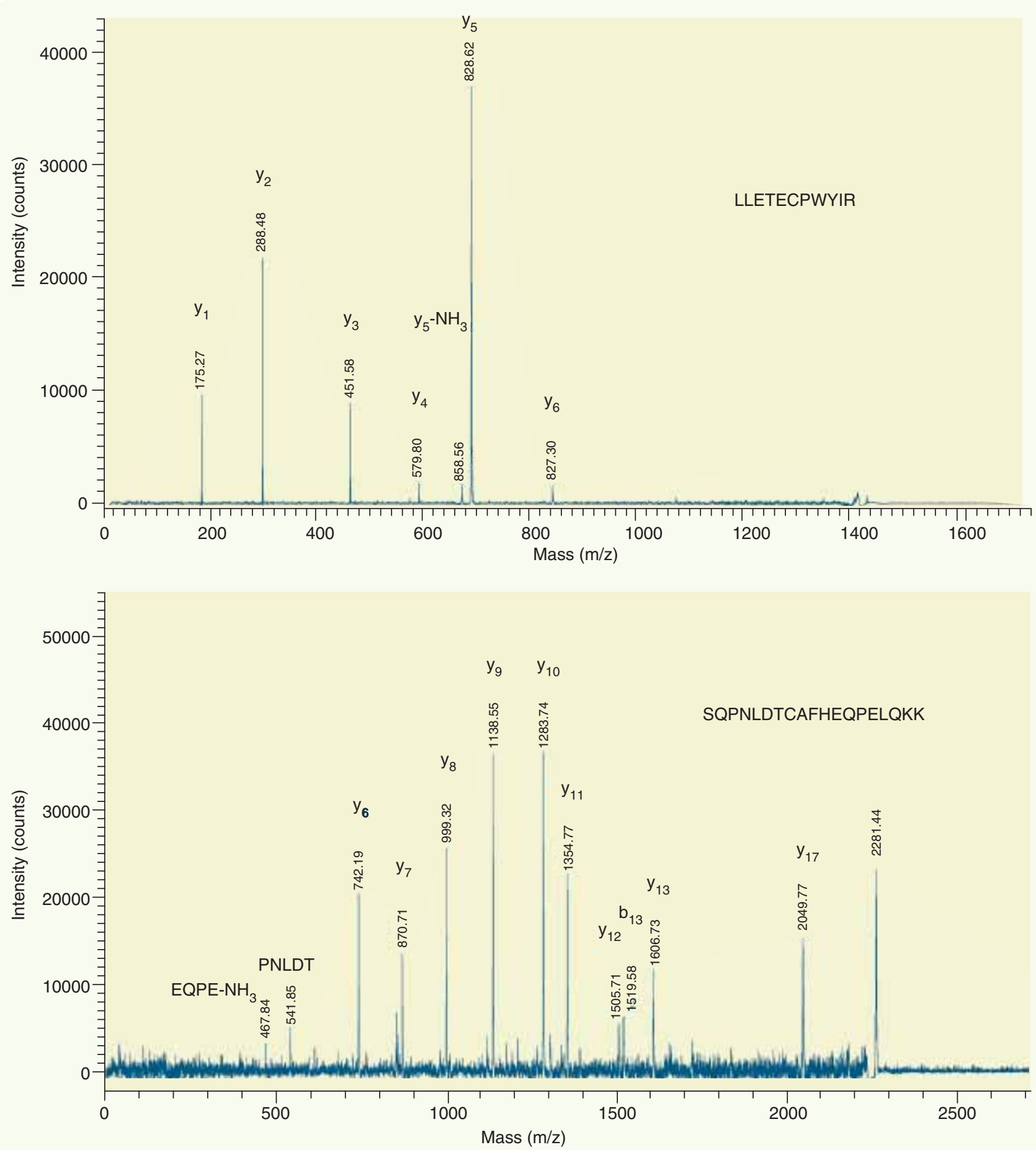

Figure 1. Automatically acquired MALDI- LIFT/PSD-MS/MS spectra of LC- separated performic acid- oxidized saliva peptides containing a cysteic acid residue. The sulfonic acid functionality of the cysteic acid enables fragmentation according to the mobile proton model. Simple fragment ion spectra displaying predominantly $y$-ion series between the cysteic acid residue and the next basic residue are recorded. The upper panel displays a fragment ion spectrum of a calgranulin A tryptic peptide, while the lower panel shows a fragment ion spectrum of a tryptic peptide from a cystatin precursor.

LC: Liquid chromatography; MALDI: Matrix-assisted laser desorption/ionization; MS/MS: Tandem mass spectrometry; m/z: Mass-to-charge ratio; PSD: Post-source decay. 


\section{Protein identification by 2DE MALDI-MS}

A combination of high-resolution $2 \mathrm{DE}[29,30]$, highly sensitive biological MS and the rapidly growing protein and DNA sequence databases has provided the basis for resolving and characterizing the components of proteome extracts from tissue samples, cells and subcellular fractions of various species including microorganisms, plants, animals and humans [28,56]. Of note, more than 30 years after its development, $2 \mathrm{DE}$ is still the only method available that can resolve several thousand protein species in a single experiment [57,58], while MS is the only technology that can unambiguously identify and further characterize low femtomole amounts of many different proteins in a short time. This is typically achieved by excising samples out of the 2D gel, in situ proteolysis of the immobilized proteins and PMM and MS/MS analyses. W ith respect to throughput and automation, M ALD I-TOF-M S in combination with PM M is the preferred mass spectrometric analysis strategy.

In 2DE, protein concentration levels are visualized with staining or labeling methods, estimated and cataloged by a high-resolution image of the 2D gel. Spot excision, in situ proteolysis and extraction of the cleavage products from the gel

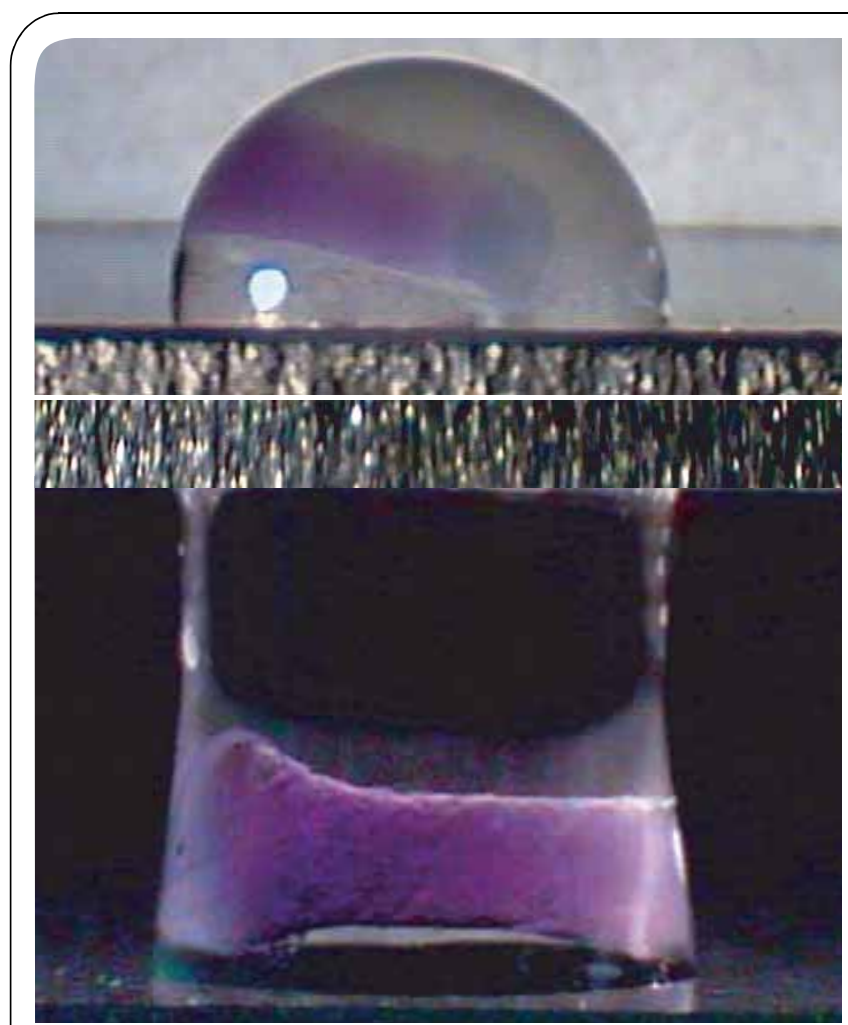

Figure 2. Photographs of insitu proteolysis from a gel piece in a 1- $\mu$ l droplet on a surface-modified chip (upper panel) and peptide isolation using a second surface-modified chip consisting of small anchors coated with a thin layer of the MALDI matrix $\alpha$-cyano-4-hydroxycinnamic acid that is in contact with the sample droplet from above, to which peptides preferentially bind (lower panel). The second chip can be directly used as a target for MALDI mass spectrometry experiments.

MALDI: Matrix-assisted laser desorption/ionization. matrix, peptide purification and concentration, and the mass spectrometric sample preparation are then the crucial steps that interface the two analytical techniques. Today, these routines, and not the mass spectrometric instrumentation, determine how many protein samples can be identified per day.

$M$ ass spectrometric identification of a few hundred protein species separated by 2D E al ready puts stress on sample preparation, analysis times and data interpretation. In order to increase the sample throughput, parallel sample processing has been implemented whenever possible. When this was not possible, the necessary serial processing routines have been automated and became fast. The excision of detected protein spots and the mass spectrometric analyses fall into the latter category, whilst into the former fall in situ proteolysis, extraction of the digestion products, peptide purification/concentration and MALD I sample preparation. In these cases, to achieve H TP, all sample processing is performed in 96- or 384-microtiter plates (MTPS). Protocols, which can easily be applied to many samples at a time, have been developed and support the downstream processes [56]. For instance, peptide purification and concentration has been integrated into the MALDI sample preparation process, and the resulting samples facilitate fully automated data acquisition [59]. This is possible as the samples are small (400-600 $\mu \mathrm{m}$ diameter) and relatively homogeneous, thereby minimizing changes of the desorption/ionization laser irradiation during data acquisition. M any of these protocols employ so-called anchor targets, which are coated with a hydrophobic layer incorporating small micrometer-sized hydrophilic anchors [60]. The M ALDI sample droplet shrinks and crystallizes on these anchors in a relatively well-defined manner, resulting in MALDI samples of the size of the anchor and concentrating the analyte. The hardware developed include automated gel processing workstations, which are now available from various manufacturers and include image acquisition, fully automated excision of the detected protein spots, and delivery of the excised fractions into 96-well MTPS. Another class of instruments that has been developed and commercialized automates the subsequent liquid handling, incubation steps and recovery of the cleavage peptides out of the gel matrix. FIGURE 2 illustrates two steps in a protocol that has been devised for automated sample preparation for MALDI-M S analysis of protein spots separated by $2 \mathrm{DE}$.

Significant changes in 2DE protein spots provide the basis for comparative studies, which can address many different questions. Typical examples are the monitoring of the composition of a protein population in a cell or the concentration levels of a number of selected protein candidates over a time course following the onset of a stress regime, stimulation or uptake of a drug. Besides fundamental studies, the combination of 2DE and M ALDI-MS has been used extensively for detection and identification of biomarkers for many different diseases, including cancer, Alzheimer's disease, allergy and inflammatory diseases. H owever, to generate meaningful results has proven difficult and laborious. M any samples and controls need to be analyzed under the same conditions, several times each, in order 
to recognize and separate experimental from biological variations. This is another reason for streamlining and automation of the techniques and protocols involved.

\section{Protein identification by liquid chromatography MALDI-MS}

In parallel to 2DE, LC is an important separation technique for proteins and peptides in proteome research $[25,26]$. While no LC separation mode al one has the resolving power of $2 \mathrm{DE}$, several different separation modes are often used in series, or in combination with gel electrophoresis, to fractionate complex proteomic extracts. Some LC separations are performed on the intact proteins (e.g., size-exclusion chromatography and ionexchange chromatography), but most are performed on peptides produced by proteolytic cleavage of the proteins. This approach relies completely on the ability to identify the proteins contained in the original sample by MS/MS analysis of the detected peptides.

Although the configurations of LC instrumentation vary greatly, the last separation step prior to MS is most often reversed phase (RP)LC. RPLC has high resolving power for peptides, provides enrichment of analytes in dilute samples, affords efficient sample desalting, and operates with aqueous-organic solvents, which makes it directly compatible with MS analysis. To optimize analyte recovery, which is often limited in proteomic applications, LC systems are used that have minimal surfaces and dead volumes, and operate at flow rates in the range of $\mathrm{nl} / \mathrm{min}$. M ost frequently, the LC eluent is infused online to ESIM S/M S (nano-RPLC/ESI-M S/M S). H owever, the development of M ALDI-M S/M S instrumentation, such as M ALDI-TOF/TO F and M ALDI-qTOF, has highlighted the technical capability for using M ALDI as an attractive and efficient alternative to ESI for LC/M S/M S of complex peptide mixtures [61].

The offline nature of the LC coupling to M ALD I offers some distinct advantages compared with ESI for the analysis of chromatographic effluent $[62,63]$. W ith LC/ESI-M S, the time available for analysis of a compound is limited to the time width of its chromatographic peak. In complex peptide mixtures, in which several analytes coelute, this time interval is often too short for acquisition of fragment ion spectra of all sample components. This time constraint does not apply to M ALDI-M S. After initial mass analysis, which only consumes a minor part of the sample, the remainder of the sample can be stored on the MALDI sample plate for several days without degradation of the analyte molecules. This allows the use of a different data acquisition strategy. Initially, all LC fractions are analyzed in M S mode. The mass spectral data of the intact peptide ions in all fractions then provide the information that guides the subsequent M S/M S analysis, thereby enabling the specific selection of particular peptides. Similarly, the information obtained by a first set of M S/M S analyses can be used to guide the selection of precursor ions for the following MS/MS analyses. In this manner, LC/M ALDI enables efficient data-dependent analyses.

$W$ ith respect to large-scale analysis, multidimensional LC has successfully been applied to whole proteome analysis [26]. Exploitation of the peptide-specific information from the LC separation, such as the retention time, can further assist protein identification [64]. In combination with accurate peptide mass measurement, it has been shown that the combined information is sufficiently powerful to analyze entire proteome extracts through one LC/M S experiment without the need to undertake M S/M S for repetitive analyses [65]. These new LC/M S analysis strategies have been demonstrated exclusively in combination with ESI. The potential contribution of MALDI-MS to these has not yet been evaluated.

\section{Affinity purification}

In general, affinity purification tries to overcome the limitations in proteomics caused by the low amount of analyte present in a sample full of contaminants and/or analytical competitors. It is used to selectively enrich the analyte of interest. This objective is obviously desirable for any subsequent analytical experiment. H owever, some of the procedures can be more suitable or acceptable for M ALD I-M S in H TP proteomics than others.

Affinity purification can be extremely powerful if high-affinity binding with proper release mechanisms can beengineered. In this context, the previously discussed peptide derivatization methods play a major role in many affinity purification strategies by labeling peptides or proteins with an affinity tag (e.g., biotin) to enable effective and selective separation (e.g., using avidin columns).

M ost of the affinity purification systems are column-based LC systems and, hence, not specifically developed for M ALD I-M S. Although much of the LC (including liquid affinity chromatography) appears to be better suited for ESI-M S analysis due to its easier direct coupling to ESI ion sources, offline sample collection and preparation for M ALD I-M S also has its advantages (see previous section). Alternatively, some of the affinity purification methods can now be performed on so-called chips, which provide a platform for miniaturized and large-scale purification. Examples are lab-on-a-chip devices [66] and surfacemodified M ALDI targets [67]. While the former usually incorporate some microfluidic sample purification, the latter depend only on the active surface area on the MALDI target using methods such as conventional sample spotting and washing via dispension and aspiration (or evaporation). The active surface areas of these chips are relatively small and thus limit the total amount of recovered analyte. Chip technology can be ideal for H TP applications if the chips are cheap or recyclable, thereby guaranteeing no sample carry-over. Thus far, MALDI affinity chips have been developed for many applications such as immobilized metal ion affinity chromatography (IMAC) and even epitope binding through antibody immobilization. H owever, all of these are expensive and often cannot be reused.

\section{Improvements of the MALDI process}

Although M ALDI-M S analysis is not currently the bottleneck in proteomics, future developments in M ALDI have the potential to enhance its analytical capabilities in the proteomic workflow through increased sensitivity or greater compatibility with up- and downstream methods. Any further improvements will inevitably also have a direct impact on the sample throughput. 
Recently, a study was published that reported the succesful detection from post-ionized high-molecular proteins and D N A from a M ALD I-generated plume. This second ion population was apparently generated in addition to the primarily formed MALDI ions [68]. This report clearly demonstrates the ionization inefficiency of the MALDI process. However, in this study, infrared-M ALD I was used, and many relatively large chunks of sample can be expected to be in the plume. $\mathrm{H}$ ence, the yield of the secondary MALDI process of the plume material is putatively higher here than in the analogous UV-MALDI case where there is less ablation and a smaller amount of bulk material in the plume. N onetheless, it will be worthwhile to determine the additional gain in the ion signal when effective UV-MALDI post-ionization is applied and all primary and secondary M ALDI-formed ions are accumulated.

Another area of fundamental importance is M ALDI sample preparation. To date, mostly solid matrices have been used, again mainly due to their superior performance with regard to sensitivity. Since only two of them are used in more than approximately $90 \%$ of the proteomics work with the analyte embedded in the crystalline matrix structure, sample manipulation and the tuning of the sample conditions (e.g., pH range or on-target sample reactions) are greatly restricted. As discussed earlier, many competitive drawbacks of M ALDI in comparison to ESI are based on the difference between solid and liquid state of the sample required for either of these two techniques. Although it is an inherent requirement for $\mathrm{ESI}$ to use liquid samples, it is not mandatory for MALDI to use solid matrices. In fact, it has even been shown by the inventors of MALDI that liquid matrices do work. H owever, until recently all liquid matrix systems lacked sensitivity, thus fuelling the wide-spread belief that the MALDI process only works effectively if the analyte is properly embedded in the matrix crystal lattice. Interestingly, just a few minor changes in the sample preparation of a known liquid matrix system proved enough to dramatically improve the analytical sensitivity, particularly in proteomic analysis, without losing any of the benefits that a liquid sample system can provide [69]. BOX 2 lists some of the many advantages a liquid MALDI sample can provide and FIGURE 3 shows a liquid M ALDI-M S spectrum of a subpicomole bovine serum albumin digest prepared according to reference [69].

These two new approaches are just examples of novel concepts that can arise from further developments in the M ALD I technique itself. Since the MALDI process is very poorly understood, there is vast potential in the future for many more fundamental improvements in M ALDI-M S.

There are other approaches that try to circumvent the use of a matrix and its disadvantages all together (e.g., matrix ion and matrix adduct ion formation and detection) [70-73]. $M$ atrix-free laser desorption/ionization such as desorption/ionization on silicon (DIOS) [71,72] or silicon nanowires [73] are examples for techniques that have been used to analyze small molecules and pure peptides, but also protein digests at levels as low as $5 \mathrm{fmol}$.

\section{MALDI-MS pattem diagnostics}

$O$ ne interesting alternative to structural or sequence analysis is the use of the accurate and precise mass spectral data as a pattern without being concerned about the ions' identity of individual peaks. These patterns can be used to identify not only different diseases and their various levels of progression [74-76], but also whole organisms such as bacteria [77-79]. The underlying idea is simple and based on the assumption that the changes in the mass spectral pattern are specific and only due to differences in the biological system and are not due to experimental variations. By creating spectral libraries or classification systems, simple recording of a mass spectrum can enable the sample's diagnosis (e.g., identification of its state and origin).

Particularly, the analysis of proteomic patterns is thought to be extremely informative since many specific biomarkers are proteins. It has been suggested that proteome mass spectra recorded from body fluids can provide effective clinical screening for disease [74-76]. This concept has been termed proteomic pattern diagnostics. Thus far, the concept and initial results have been presented. H owever, there is an extensive debate taking place, discussing the published data and the general feasibility of this approach [80-84]. The main focal points of this debate are the difficulties in the detection of specific and usually low-abundant biomarkers in a sea of abundant proteins

\section{Box 2. Liquid MALDI characteristics and their impact on mass spectrometric analysis.}

- Homogeneous sample $(A) \rightarrow$ stable ion flux and signal $\rightarrow$ facilitates automation, quantification and coupling to FTCR-ion trap MS (B) $\rightarrow$ desorption spot and laser parameter changes are unnecessary $\rightarrow$ less sample consumption and faster analysis

- MALDI directly from liquid delivery systems $\rightarrow$ online LC coupling and continuous acquisition

- Greater flexibility of matrix systems $\rightarrow$ greater analyte versatility

- New potential applications such as online reaction monitoring

- No inherent purification or concentration as in solid MALDI through the crystallization process, but concentration via evaporation of volatile solvent components and tolerance to contaminants is still achievable

- Liquid MALDI-MS is approaching the sensitivity level of solid MALDI-MS

FTCR: Fourier transform ion cyclotron resonance; LC: Liquid chromatography; MADLI: Matrix-assisted laser desorption/ionization; MS: Mass spectrometry. 


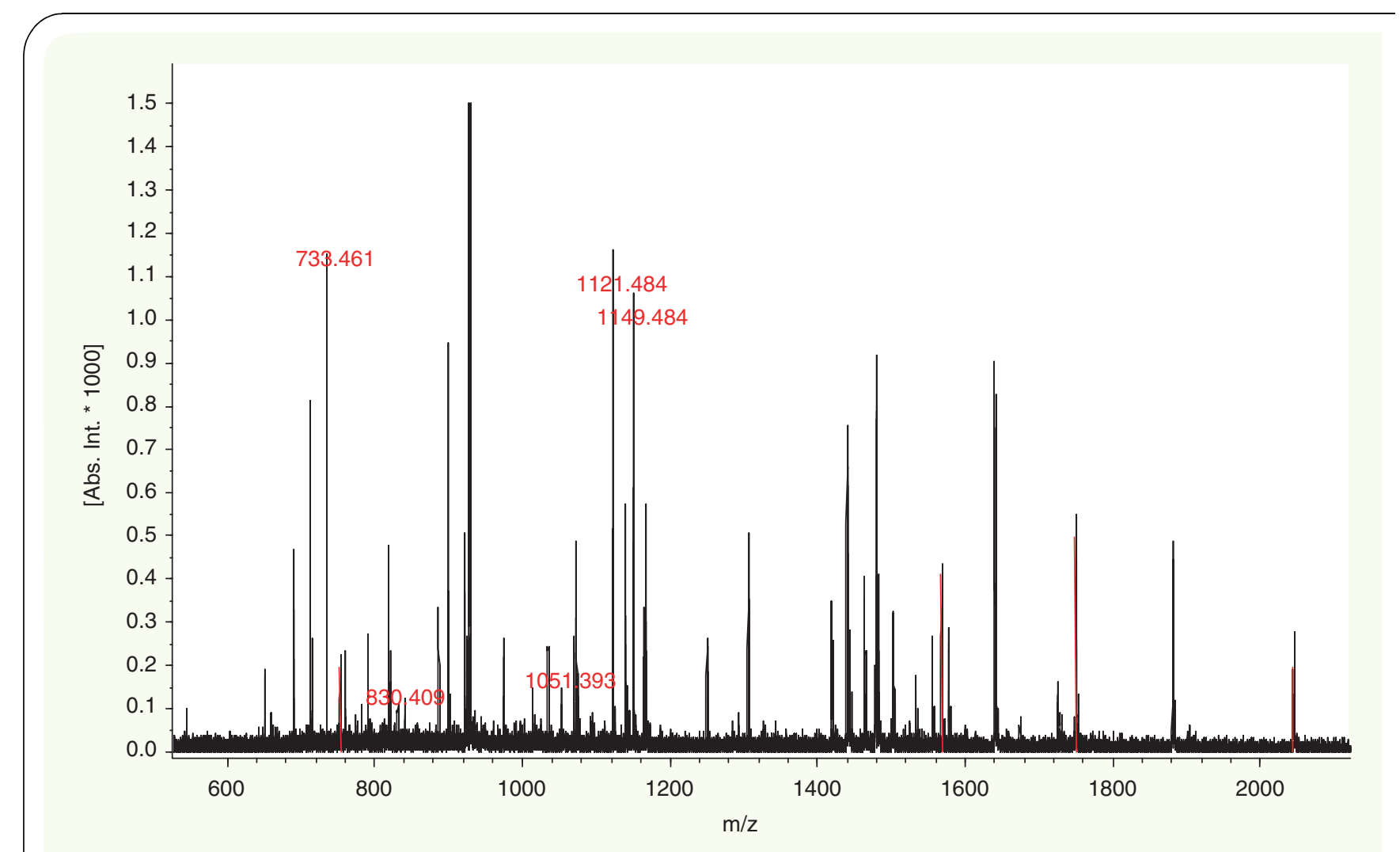

Figure 3. Liquid ultraviolet matrix-assisted laser desorption/ionization mass spectrum of a $\mathbf{5 0}$ - fmol bovine serum albumin digest. Automated peak labeling and peptide mass mapping resulted in 39 matched peaks and only five unmatched peaks (labeled with $\mathrm{m} / \mathrm{z}$ values in spectrum) giving a sequence coverage of $65 \%$.

and the reliability of the methodology for robust clinical screening. $N$ aturally, even more so than in protein identification strategies, variations in the laboratories and protocols can highly influence the outcome of this type of analysis, which suggests that differences are potentially due to artifacts and are not disease specific. To date, surface-enhanced laser desorption/ionization (SELDI) is the most extensively employed method for proteomic pattern diagnostics. SELDI is a proprietary technology based on surface-modified MALDI target plates, which provide on-target affinity purification. Particularly with regard to SELDI, the merits and drawbacks of the technology and its associated mass spectrometric readout for clinical screening have been heavily discussed [76].

$N$ onetheless, if these obstacles can be overcome and a reliable methodology can be developed, proteomic pattern diagnostics would be the most significant advance in clinical screening. And again, due to its ease, speed, robustness and low cost, M ALDIMS would be the technology of choice, particularly if greater ion signal stability as in liquid M ALD I-M S can be achieved.

\section{Imaging MS}

MALDI imaging MS is an emerging technique in proteomics that allows the analysis of the spatial distribution of many peptides and proteins within a tissue sample simultaneously [85,86]. Sample preparation includes either a blot of the tissue of interest transferred onto a hydrophobic surface [85] or a frozen thin $(5-20 \mu \mathrm{m})$ section of it [87]. Either way, the sample is immobilized on a M ALDI sample plate and coated with a thin film of M ALDI matrix. Typically, M ALDI-TOF instruments are used to acquire position-correlated mass spectra in a Cartesian pattern that covers the area of interest. Analogous to a picture produced by an optical color scanner consisting of pixels with intensity values for different colors, the mass spectrometric image consists of pixels, each represented by one mass spectrum, with ion signal intensities for $\mathrm{m} / \mathrm{z}$ values. By assigning different colors to selected $\mathrm{m} / \mathrm{z}$ values, the mass spectrometric image can be used to visualize the spatial distribution of the associated compounds in the studied tissue.

M ALD I-TOF imaging M S has been applied to various tissue sections including diseased samples from human gliomas and non-small cell lung cancer [86]. In-depth analysis of the recorded mass spectrometric images revealed both disease state and patient prognosis protein patterns. A hierarchical cluster analysis showed that M ALDI imaging M S can distinguish subgroups of tumor tissue from each other as well as from nontumor tissue.

The spatial resolution of MALDI imaging M S is limited by the spot size irradiated for ion formation, which on most currently installed instruments falls between 50 and $150 \mu \mathrm{m}$, and therefore excludes cellular imaging. $\mathrm{H}$ owever, it has been shown that MALDI imaging MS with a spatial resolution of $1 \mu \mathrm{m}$ is 
possible if the hardware is optimized for this task [88]. The main drawback of smaller desorption spot sizes is that significantly fewer molecular ions are generated per laser pulse, leading to significantly reduced detection sensitivity. In addition, since the image is recorded sequentially (pixel by pixel), the total analysis time increases drastically, as does the raw data volume. In automated mode, state-of-the-art M ALD I-TOF mass spectrometers can analyze several sample spots (positions) per second, enabling the acquisition of MALDI-MS images that comprise many thousand position-correlated mass spectra in a few hours. It is foreseeable that the next generation of MALDI-TOF instruments will afford even shorter analysis times, which makes the use of M ALDI imaging M S even more attractive.

A different approach, termed M ALD I-M S imaging in microscope mode, which shortens the total analysis time drastically, was recently demonstrated [89]. Compared with optical methods, this technique operates by the principle of a microscope rather than a laser scanner. Instead of many small spots (pixels), a relatively large area is simultan eously irradiated and the generated ion cloud, instead of being focused, is widened (ion microscope) before the ions pass through the TO F analyzer and produce an ion-optical image on a position-sensitive detector (array ion detector). It was shown that from a $200 \mu \mathrm{m}$ sample, spot peptide molecular ion images with a lateral resolution of $4 \mu \mathrm{m}$ could be recorded.

With regard to lateral resolution, it must be noted that the sample preparation naturally determines the quality and resolution of the mass spectrometric image. For instance, if the average lateral migration of the peptides and proteins during solubilization by the matrix solution and incorporation into the matrix crystals during solvent evaporation exceeds $10 \mu \mathrm{m}$, an image resolution of $5 \mu \mathrm{m}$ is obviously unnecessary.

Although falling outside the scope of this review, it should be mentioned that the combination of MALDI sample preparation techniques with secondary ion MS (SIMS), termed matrix-enhanced (M E)-SIM S, has recently gained attention as an alternative technique for imaging $\mathrm{M} \mathrm{S}[90,91]$. It was shown that the M ALD I matrix 2,5-dihydroxybenzoic acid enables sensitive SIM S analysis of peptides $(<2.5 \mathrm{kD}$ a) and that imaging of nervous tissue at subcellular resolution is feasible. O ne attractive advantage of SIMS over MALDI-MS is its high lateral resolving power $(<1 \mu \mathrm{m})$. H owever, the analysis times per spot (pixel) are significantly longer (minutes) compared with M ALDI-TOF-M S $(<1 \mathrm{~s})$. In these studies, the matrix solution was electrosprayed onto the tissue section and it could be shown that this treatment preserves the spatial distribution of surface analytes in the analyzed tissue section at a level of approximately $3 \mu \mathrm{m}$.

\section{Summary \& conclusion}

The main technical advances in M S such as the introduction of high-sensitivity, speed and performance mass spectrometric equipment have been made. H owever, there are many more areas in proteomics that are crucial for successful proteomewide HTP analyses. Although not discussed in this review, bioinformatics still has to accelerate further to fully exploit the flood of data that one experiment can provide as well as the data that are now available from the experimental results of the worldwide collective of scientists and their research efforts. 0 ne other aspect is the enormous data flux itself. To be truly capable of H TP proteomics, adequate arrangements have to be made to cope with the data acquisition rate and the data storage and processing. None of these areas have been adequately addressed yet. The preparation of proteome samples is by far the most daunting exercise. Here lies the main stumbling block for proteomics and consequently its HTP analytical capabilities. Proteomics is a relatively new field and, although many sky-high promises have been made for the immediate future, only a few strategies and laboratories are currently capable of analyzing proteomes on a scale that might be considered as large and rapid, the attributes characterizing H TP. H ence, the immediate future will still be occupied by developing further improvements in proteomic technologies and it is the authors' conviction that proteomics, together with its H TP application, is still in its infancy. Whether M ALDI-M S will be the center point in any of the future H TP proteomic strategies remains to be seen. Currently, with its potential as described in this review, it is the most promising candidate to deliver the promises that have been made.

\section{Expertcommentary}

It has recently been claimed that new advances in M ALDI-M S technology now facilitate the identification of 30,000 proteins per day using a single instrument (i.e., one protein identified every $3 \mathrm{~s}$ ). This number of protein identifications would theoretically satisfy the size of the human proteome given that there is one gene product per gene. O bviously, claims like this have put expectations on proteomics that are not, and probably never will be, achievable. Regrettably, this is damaging to the serious efforts of researchers to advance the throughput in proteomics.

$\mathrm{N}$ evertheless, it is clear that proteomics (i.e., protein analysis) is needed to elucidate the working principles of cells and whole organisms. Unfortunately, the analysis of proteins is far more complex than the analysis of DNA or RN A and, in addition, it is hampered by the scarcity of its subjects. This is a particular problem for HTP analysis since flexibility and intelligent decision making at any point in the analytical process pose extremely high demands on any automated system and are best avoided.

$\mathrm{H}$ owever, it is believed that for specific applications such as in clinical screening programs or the analysis of specific subsets of the proteome, H TP proteomics will be possible to a certain degree and tremendously increase the knowledge base in the biomedical sciences on a much faster time scale than previously possible. M ALD I-M S will certainly play a major role in these strategies given its current standing, its unexplored potential and the lack of any serious alternative to biological M S as a sensitive and fast analytical tool in proteomic research.

And finally, one of the most important goals for all future developments in HTP proteomics must be the increase of informative data, not just data. It is easy to increase the sample 
throughput and the analytical data sets but often this is only achieved by acquiring redundant or even useless data. This needs to be avoided under any circumstances if proteomics is to effectively contribute to postgenomic understanding in the biomedical sciences.

\section{Five-year view}

The authors' 5-year view is similar to the one recently published by Q ian and coworkers [65]. M ore specifically, new and significant developments in the areas of peptide/protein sample preparation for MALDI-MS and the MALDI technique itself are anticipated. The former will be driven by the need to provide higher sensitivity for the problematic cases such as posttranslationally modified, membrane and generally low-abundant proteins. Here, effective derivatization of peptides and proteins will play a key role. Once these new advances have been established, their implementation in an automated robotic workflow will be the next step. These two processes are obviously highly interconnected and will be influenced by the feasibility of the developed procedures in both.

It is also believed that new breakthroughs in the MALD I technique itself will provide novel concepts to some of the problem areas in MALDI-M S-based HTP proteomics. For instance, liquid M ALDI can provide an entirely new method of performing M ALD I-M S analysis, eliminating the disadvantages that exist in comparison with ESI-M S. It is also well known for MALDI that the efficiency of producing molecular ions compared with the overall sample consumption is generally fairly poor. In the next 5 years, new M ALDI methods to improve this efficiency will almost certainly be identified.

\section{Acknowledgements}

The authors thank Richard Jacob for the acquisition of the spectra in FIGURE 1.

\section{Key issues}

- Effective peptide and protein sample preparation (including separation, purification and derivatization) that can be automated plays a major role in high-throughput proteomics.

- Matrix-assisted laser desorption/ionization (MALDI) is an ionization technique that is poorly understood. Thus, there is scope for improvements and new developments in MALDI, which can also be beneficial for proteomics. Examples for potential areas of development are peptide derivatization, liquid MALDI mass spectrometry (MS) and MALDI post-ionization.

- MALDI-MS is easy to implement and is a reliable technology. It can be relatively inexpensive and extremely fast with regard to data acquisition. Hence, it is ideal for large-scale applications such as clinical screening programs.

- For optimal data exploitation and high-throughput proteomics, it is essential to develop advanced bioinformatic tools for data management and analysis.

\section{References}

Papers of special note have been highlighted as: - of interest

-. of considerable interest

-1 M cLuckey SA, Wells JM . M ass analysis at the advent of the 21st century. Chem. Rev. 101, 571-606 (2001).

-2 M artin RL, Brancia FL. Analysis of high mass peptides using a novel matrix-assisted laser desorption/ionisation quadrupole ion trap time-of-flight mass spectrometer. Rapid Commun. M ass Spectrom. 17, 1358-1365 (2003).

- 3 D ouglas DJ, Frank AJ, M ao D. Linear ion traps in mass spectrometry. M ass Spectrom. Rev. 24, 1-29 (2005).

$\rightarrow 4$ Ackloo S, Loboda A. Applications of a matrix-assisted laser desorption/ionization orthogonal time-of-flight mass spectrometer. I. M etastable decay and collision-induced dissociation for sequencing peptides. Rapid Commun. M ass Spectrom. 19, 213-220 (2005).

5 Syka JE, M arto JA, Bai D L et al. N ovel linear quadrupoleion trap/FT mass spectrometer: performance characterization and use in the comparative analysis of histone $\mathrm{H} 3$ post-translational modifications. J. Proteome Res 3, 621-626 (2004).

Aebersold R, M ann M . M ass spectrometrybased proteomics. N ature 422, 198-207 (2003).

- $7 \mathrm{M}$ ann M, H endrickson RC, Pandey A. Analysis of proteins and proteomes by mass 13 spectrometry. Ann. Rev. Biochem. 70, 437-473 (2001).

- Comprehensive overview on mass spectrometric technologies used in proteomics.

-8 Blueggel $\mathrm{M}$, Chamrad D, M eyer $\mathrm{HE}$. Bioinformatics in proteomics. Curr. Pharm. Biotechnol. 5, 79-88 (2004).

$\checkmark 9$ Pedrioli PG, EngJK, H ubley R et al. A common open representation of mass spectrometry data and its application to proteomics research. N ature Biotechnol. 22, 1459-1466 (2004).

- 10 Boguski M S, M clntosh M W. Biomedical informatics for proteomics. N ature 422 , 233-237 (2003)
Patterson SD . D ata anal ysis - the Achilles heel of proteomics. N ature Biotechnol. 21, 221-222 (2003).

12 Kearney $P$, T hibault P. Bioinformatics meets proteomics - bridging the gap between mass spectrometry data anal ysis and cell biology. J. Bioinform. Comput. Biol. 1, 183-200 (2003).

Fenyo D, Beavis RC . Informatics and data management in proteomics. Trends Biotechnol. 20, S35-S38 (2002).

14 H ancock W S, Wu SL, Stanley RR, Gombocz EA. Publishing large proteome datasets: scientific policy meets emerging technologies. Trends Bi otechnol. 20, S39-S44 (2002).

15 D reisewerd $K$. The desorption process in M ALDI. Chem. Rev. 103, 395-426 (2003).

- Extensive review of the fundamentals of the desorption process in matrix-assisted laser desorption/ionization (M ALDI).

16 Karas M, Kruger R. Ion formation in M ALDI: the cluster ionization mechanism. Chem. Rev. 103, 427-440 (2003). 
17 Knochenmuss R, Zenobi R. M ALDI ionization: the role of in-plume processes. Chem. Rev. 103, 441-452 (2003).

- [16] and [17] are reviews providing basic ideas for the understanding of the M ALD I ionization process.

18 Karas M, Hillenkamp F. Laser desorption ionization of proteins with molecular masses exceeding 10,000 daltons. Anal. Chem. 60, 2299-2301 (1988).

-19 Rappsilber J, M oniattes M , N ielsen M L, Podtelejnikov AV, M ann M. Experiences and perspectives of M ALDI M S and M S/M S in proteomic research. Int. J. M ass Spectrom. 226, 223-237 (2003).

20 M amyrin BA. Time-of-flight mass spectrometry (concepts, achievements, and prospects). Int. J. M ass Spectrom. 206, 251-266 (2001).

21 Cech N B, Enke CG. Practical implications of some recent studies in electrospray ionization fundamentals. M ass Spectrom. Rev. 20, 362-387 (2001).

22 Fenn JB, M ann M, M eng CK, Wong SF, W hitehouse $C M$. Electrospray ionization for mass spectrometry of large biomolecules. Science 246, 64- 71 (1989).

-23 Tang K, Page JS, Smith RD. Charge competition and the linear dynamic range of detection in electrospray ionization mass spectrometry. J. Am. Soc. M ass Spectrom. 15, 1416-1423 (2004).

$\checkmark 24$ Steen H, Jebanathirajah JA, Springer M, Kirschner M W. Stable isotope-free relative and absolute quantitation of protein phosphorylation stoichiometry by M S. Proc. N atl Acad. Sci. U SA 102, 3948- 3953 (2005).

-25 Tomer KB. Separations combined with mass spectrometry. Chem. Rev. 101, 297-328 (2001)

-26 Washburn M P, Wolters D, Yates JR III. Large-scale analysis of the yeast proteome by multidimensional protein identification technology. N ature Biotechnol. 19, 242-247 (2001).

-27 Pappin DJC, H ojrup P, Bleasby AJ. Rapid identification of proteins by peptide-mass fingerprinting. Curr. Biol. 3, 327-332 (1993).

28 N aaby- $\mathrm{H}$ ansen S, Waterfield M D , C ramer R. Proteomics - post-genomic cartography to understand gene function. Trends P harmacol. Sci. 22, 376-384 (2001).

290 'Farrell PH. High resolution twodimensional electrophoresis of proteins. J. Biol. Chem. 250, 4007-4021 (1975).

- 30 Klose J. Protein mapping by combined isoelectric focusing and electrophoresis of mouse tissues. A novel approach to testing $\nabla_{4} \mathrm{O}$ da Y, Huang K, Cross FR, Cowburn D, for induced point mutations in mammals. H umangenetik 26, 231-243 (1975).

31 Chaurand P, Luetzenkirchen F, Spengler B. Peptide and protein identification by matrix-assisted laser desorption ionization (M ALDI) and M ALDI-post-source decay time-of-flight mass spectrometry. J. Am. Soc. M ass Spectrom. 10, 91-103 (1999).

- 32 Spengler B. Post-source decay analysis in matrix-assisted laser desorption/ionization mass spectrometry of biomolecules. J. M ass Spectrom. 32, 1019-1036 (1997).

33 Steen $H, M$ ann M. The ABC's (and $X Y Z$ 's) of peptide sequencing. N ature Rev. Mol. Cell Biol. 5, 699-711 (2004).

- 34 Yergey AL, C oorssen JR, Backlund PS Jr et al. D e novo sequencing of peptides using M ALDI/TOF-TOF. J. Am. Soc. M ass Spectrom. 13, 784-791 (2002).

-35 Bienvenut W V, D eon C, Pasquarello C et al. $M$ atrix-assisted laser desorption/ionization-tandem mass spectrometry with high resolution and sensitivity for identification and characterization of proteins. Proteomics 2 , 868-876 (2002).

- [34] and [35] are the first articles reporting the design and application of M ALD I time-of-flight (TOF)/TOF tandem mass spectrometry (M S/M S).

- 36 Suckau D, Resemann A, Schuerenberg M, $H$ ufnagel $P$, Franzen J, H olle A. A novel M ALDI LIFT-TOF/TOF mass spectrometer for proteomics. Anal. Bioanal. Chem. 376, 952-965 (2003).

Shevchenko A, Loboda A, Ens W, Standing KG. M ALD I quadrupole time-of-flight mass spectrometry: a powerful tool for proteomic research. Anal. Chem. 72, 2132-2141 (2000).

38 K rutchinsky AN, Z hang W, Chait BT. Rapidly switchable matrix-assisted laser desorption/ionization and electrospray quadrupole-time-of-flight mass spectrometry for protein identification. J. Am. Soc. M ass Spectrom. 11, 493-504 (2000).

39 K rutchinsky AN, Kalkum M, Chait BT. Automatic identification of proteins with a M ALD I-quadrupole ion trap mass spectrometer. Anal. Chem. 73, 5066-5077 (2001).

-40 O ng SE, Foster LJ, M ann M. M ass spectrometric-based approaches in quantitative proteomics. M ethods 29, 124-130 (2003).

-41 M oritz B, M eyer H E. Approaches for the quantification of protein concentration ratios. Proteomics 3, 2208-2220 (2003).
$C$ hait $B T$. Accurate quantitation of protein expression and site-specific

phosphorylation. Proc. N atl Acad. Sci. U SA 96, 6591-6596 (1999).

43 Pratt JM, Petty J, Riba-Garcial et al. D ynamics of protein turnover, a missing dimension in proteomics. $M$ ol. Cell Proteomics 1, 579-591 (2002).

- A method for determination of protein half-lives based on metabolic stable isotope labeling.

44 Ross PL, H uang YN, M archese JN et al. $M$ ultiplexed protein quantitation in Saccharomyces cerevi siae using amine-reactive isobaric tagging reagents. M ol. Cell. Proteomics 3, 1154-1169 (2004).

- D emonstration of facile multiplexed protein quantitation using $\mathbf{M ~} \mathbf{s} / \mathbf{M ~ S}$.

-45 Gygi SP, Rist B, Gerber SA, Turecek F, Gelb M H, Aebersold R. Q uantitative analysis of complex protein mixtures using isotope-coded affinity tags. $\mathrm{N}$ ature Biotechnol. 17, 994-999 (1999).

- $\quad$ First article demonstrating isotope labeling for global protein quantification in $\mathbf{M ~ S - b a s e d ~ p r o t e o m i c s . ~}$

-46 Tao WA, Aebersold R. Advances in quantitative proteomics via stable isotope tagging and mass spectrometry. Curr. 0 pin. Biotechnol. 14, 110-118 (2003).

47 Brancia FL, M ontgomery H , Tanaka K, Kumashiro S. Guanidino labeling derivatization strategy for global characterization of peptide mixtures by liquid chromatography matrix-assisted laser desorption/ionization mass spectrometry. Anal. Chem. 76, 2748-2755 (2004).

-48 Beardsley RL, Reilly JP. O ptimization of guanidination procedures for M ALD I mass mapping. Anal. Chem. 74, 1884-1890 (2002).

-49 Stewart N A, Pham VT, Choma CT, Kaplan $\mathrm{H}$. Improved peptide detection with matrix-assisted laser desorption/ionization mass spectrometry by trimethylation of amino groups. Rapid Commun. M ass Spectrom. 16, 1448-1453 (2002).

-50 Pashkova A, M oskovets E, Karger BL. Coumarin tags for improved analysis of peptides by M ALD I-TO F M S and M S/M S. 1. Enhancement in M ALDI MS signal intensities. Anal. Chem. 76, 4550-4557 (2004).

- $\quad \mathrm{New}$ derivatization approach to improve peptide ionization efficiency in M ALD I-M S. 
51 Keough T, Youngquist RS, Lacey M P. Sulfonic acid derivatives for peptide sequencing by M ALDI M S. Anal. Chem. 75, 156A-165A (2003).

- O verview of the mobile proton theory and its exploitation through chemical peptide derivatization for improving peptide sequencing by M ALD I-M S/M S.

-52 Hellman U, Bhikhabhai R. Easy amino acid sequencing of sulfonated peptides using post-source decay on a matrix-assisted laser desorption/ionization time-of-flight mass spectrometer equipped with a variable voltage reflector. Rapid Commun. $M$ ass Spectrom. 16, 1851-1859 (2002).

- 53 M arekov LN, Steinert PM. Charge derivatization by 4-sulfophenyl isothiocyanate en hancespeptide sequencing by post-source decay matrix-assisted laser desorption/ionization time-of-flight mass spectrometry. J. M ass Spectrom. 38, 373-377 (2003).

- 54 Wang D, Kalb SR, Cotter RJ . Improved procedures for $\mathrm{N}$-terminal sulfonation of peptides for matrix-assisted laser desorption/ionization post-source decay peptide sequencing. Rapid Commun. M ass Spectrom. 18, 96-102 (2004).

55 Thompson AJ, Calnan D, Cramer R Cysteic acid-mediated proteome analysis: selective generation and isolation of cysteic acid peptides for mass spectrometric sequencing. In: ASM S Conference on M ass Spectrometry; T N , U SA. ThO Cam 10, 35 (2004).

- [52-55] demonstrate enhanced M ALD I peptide sequencing by sulfonation

- 56 N ordhoff E, Egelhofer V, G iavalisco P et al. Large-gel two-dimensional electrophoresis-matrix assisted laser desorption/ionization-time of flight-mass spectrometry: an analytical challenge for studying complex protein mixtures. Electrophoresis 22, 2844-2855 (2001).

- 0 verview of the workflow connecting 2D -68 gel electrophoresis and M ALD I-T OF-M S.

57 Gauss C, Kalkum M, Lowe M, Lehrach H Klose J. Analysis of the mouse proteome. (I) Brain proteins: separation by two-dimensional electrophoresis and identification by mass spectrometry and genetic variation. Electrophoresis 20, 575-600 (1999).

- 58 Rabilloud T. Two-dimensional gel electrophoresis in proteomics: old, old fashioned, but it still climbs up the mountains. Proteomics 2, 3-10 (2002).

- 59 Gobom J, Schuerenberg M, M ueller M , Theiss $D$, Lehrach $H$, N ordhoff $E$. $\alpha$ cyano-4-hydroxycinnamic acid affinity sample preparation. A protocol for

M ALD I-M S peptide analysis in proteomics. Anal. Chem. 73, 434-438 (2001).

-60 Schuerenberg $M$, Luebbert $C$, Eickhoff $H$, Kalkum M , Lehrach $H$, N ordhoff E. Prestructured M ALD I-M S sample supports. Anal. Chem. 72, 3436-3442 (2000).

-61 Zhang N, Li N, Li L. Liquid chromatography M ALD I M S/M S for membrane proteome analysis. J. Proteome Res. 3, 719-727 (2004).

- 62 M irgorodskaya E, Braeuer C, Fucini P, Lehrach $\mathrm{H}, \mathrm{G}$ obom J. N anoflow liquid chromatography coupled to matrix-assisted $>73$ laser desorption/ionization mass spectrometry: sample preparation, data analysis, and application to the analysis of complex peptide mixtures. Proteomics 5 , 399-408 (2005).

63 Ericson C, Phung QT, H orn D M et al. An automated noncontact deposition interface for liquid chromatography matrix- 75 assisted laser desorption/ionization mass spectrometry. Anal. Chem. 75, 2309-2315 (2003).

- 64 K rokhin OV, Craig R, Spicer V et al. An improved model for prediction of retention times of tryptic peptides in ion pair reversed-phase H PLC. M ol. Cell. Proteomics 3, 908-919 (2004).

65 Qian W-J, Camp D G, II, Smith RD. $\mathrm{H}$ igh-throughput proteomics using Fourier transform ion cyclotron resonance mass spectrometry. Expert Rev. Proteomics 1, 87-95 (2004).

66 Figeys D. Adapting arrays and lab-on-achip technology for proteomics. Proteomics 2, 373-382 (2002).

67 Weinberger SR, M orris TS, Pawlak M . Recent trends in protein biochip technology. Pharmacogenomics 1, 395-416 (2000).

Leisner A, Rohlfing A, Berkenkamp S, H illenkamp F, D reisewerd K. Infrared laser post-ionization of large biomolecules from an IR-M ALD (I) plume. J. Am. Soc. M ass Spectrom. 15, 934-941 (2004)

- Basic research on the M ALD I process revealing new ways to optimize its analytical sensitivity.

-69 Cramer R, Corless S. Liquid ultraviolet matrix-assisted laser desorption/ionization - mass spectrometry for automated proteomic analysis. Proteomics 5, 360-370 (2005).

- Low femtomole sensitivity of liquid ultraviolet-M ALD I peptide mass mapping providing high confidence level in protein identification.
- 70 Chen CT, Chen YC. D esorption/ionization mass spectrometry on nanocrystalline titania sol-gel-deposited films. Rapid Commun. M ass Spectrom. 18, 1956- 1964 (2004).

71 Go EP, Prenni JE, Wei J et al. D esorption/ionization on silicon time-offlight/time-of-flight mass spectrometry. Anal. Chem. 75, 2504-2506 (2003).

- 72 Thomas JJ, Shen Z, Crowell JE, Finn M G Siuzdak G. Desorption/ionization on silicon (DIO S): a diverse mass spectrometry platform for protein characterization. Proc. Natl Acad. Sci. U SA 98, 4932-4937 (2001).

Go EP, Apon JV, Luo G et al. $D$ esorption/ionization on silicon nanowires. Anal. Chem. 77, 1641-1646 (2005).

74 Rosenblatt KP, Bryant-Greenwood P, Killian JK et al. Serum proteomicsin cancer diagnosis and management. Ann. Rev. M ed. 55, 97-112 (2004).

5 Conrads TP, Zhou M, Petricoin EF III, Liotta L, Veenstra TD. Cancer diagnosis using proteomic patterns. Expert Rev. $\mathrm{Mol}$. Diagn. 3, 411-420 (2003).

76 Cottingham K. Clinical proteomics: are we there yet? Anal. Chem. 75, 472A-476A (2003).

- Critical account of proteomic pattern diagnostics.

77 Bright JJ, Claydon M A, Soufian M, Gordon DB. Rapid typing of bacteria using matrix-assisted laser desorption ionisation time-of-flight mass spectrometry and pattern recognition software. J. M icrobiol. M ethods 48, 127-138 (2002).

- 78 Fenselau C, D emirev PA. C haracterization of intact microorganisms by M ALD I mass spectrometry. M ass Spectrom. Rev. 20, 157-171 (2001).

- 79 Arnold RJ, Reilly JP. Fingerprint matching of $E$. coli strains with matrix-assisted laser desorption/ionization time-of-flight mass spectrometry of whole cells using a modified correlation approach. Rapid Commun. M ass Spectrom. 12, 630-636 (1998).

80 Stattin P, H akama M. Serum protein fingerprinting coupled with a patternmatching algorithm distinguishes prostate cancer from benign prostate hyperplasia and healthy men. Cancer Res. 62 , 3609-3614 (2002).

81 Diamandis EP. Point: proteomic patterns in biological fluids: do they represent the future of cancer diagnostics? Clin. Chem. 49, 1272-1275 (2003). 
82 Rockhill B. Proteomic patterns in serum and identification of ovarian cancer. Lancet 360, 169; author reply 170-161 (2002).

-83 Pearl DC. Proteomic patterns in serum and identification of ovarian cancer. Lancet 360, 169-170; author reply 170-161 (2002).

84 Diamandis EP. Proteomic patterns in serum and identification of ovarian cancer. Lancet 360, 170; author reply 170-171 (2002)

-85 Caprioli RM, Farmer TB, Gile J. M olecular imaging of biological samples: localization of peptides and proteinsusing M ALDI-TO F M S. Anal. Chem. 69, 4751-4760 (1997).

-86 Chaurand P, Schwartz SA, Caprioli RM . Assessing protein patterns in disease using imaging mass spectrometry. J. Proteome Res. 3, 245-252 (2004).

- Summary of recent developments in M ALD I imaging MS.

87 Stoeckli M, Chaurand P, H allahan DE, C aprioli RM . Imaging mass spectrometry: a new technology for the analysis of protein expression in mammalian tissues. $\mathrm{N}$ ature M ed. 7, 493- 496 (2001).

88 Spengler B, H ubert M. Scanning microprobe matrix-assisted laser desorption ionization (SM ALDI) mass spectrometry: instrumentation for sub-micrometer resolved LDI and M ALD I surface analysis. J. Am. Soc. M ass Spectrom. 13, 735-748 (2002).

- 89 Luxembourg SL, M ize TH, M cD onnell LA, $H$ eeren RM . H igh-spatial resolution mass spectrometric imaging of peptide and protein distributions on a surface. Anal. Chem. 76, 5339-5344 (2004).

-90 Altelaar AF, van M innen J, Jimenez CR, $H$ eeren RM , Piersma SR. D irect molecular imaging of Lymnaea stagnalis nervous tissue at subcellular spatial resolution by mass spectrometry. Anal. Chem. 77, 735-741 (2005).

- First demonstration of ion microscope imaging using M ALD I-M S.

91 M CD onnell LA, Piersma SR, $M$ aartenAltelaar AF et al. Subcellular imaging mass spectrometry of brain tissue. J. M ass Spectrom. 40, 160-168 (2005).

\section{Affiliations}

- Rainer Cramer, PhD $D$ irector, The BioC entre, Professor of M ass Spectrometry \& Bioanalytical Sciences, School of Chemistry, U niversity of Reading, Whiteknights, PO Box 221, Reading RG 6 6AS, U K Tel.: +44 1183784552 Fax: +44 1183784551 r.k.cramer@rdg.ac.uk

- Johan Gobom, PhD M ax-Planck Institute for M olecular Genetics, Innestrasse 73, 14195 Berlin, Germany Tel.: +493084131542

Fax: +49 3084131139 gobom@molgen.mpg.de

- Eckhard N ordhoff, PhD M ax-Planck Institute for M olecular Genetics, Ihnestrasse 73, 14195 Berlin, Germany Tel.: +49 3084131542 Fax: +49 3084131139 nordhoff@molgen.mpg.de 